University of Nebraska - Lincoln

DigitalCommons@University of Nebraska - Lincoln

June 2001

\title{
Cooperative freezing in spin glasses and magnetic nanostructures
}

\author{
Ralph Skomski \\ University of Nebraska-Lincoln, rskomski2@unl.edu \\ Diandra Leslie-Pelecky \\ University of Nebraska -- Lincoln, diandra2@unl.edu
}

Follow this and additional works at: https://digitalcommons.unl.edu/physicslesliepelecky

Part of the Physics Commons

Skomski, Ralph and Leslie-Pelecky, Diandra, "Cooperative freezing in spin glasses and magnetic nanostructures" (2001). Diandra Leslie-Pelecky Publications. 8.

https://digitalcommons.unl.edu/physicslesliepelecky/8

This Article is brought to you for free and open access by the Research Papers in Physics and Astronomy at DigitalCommons@University of Nebraska - Lincoln. It has been accepted for inclusion in Diandra Leslie-Pelecky Publications by an authorized administrator of DigitalCommons@University of Nebraska - Lincoln. 


\title{
Cooperative freezing in spin glasses and magnetic nanostructures
}

\author{
Ralph Skomskia) and D. Leslie-Pelecky \\ Department of Physics and Astronomy and Center for Materials Research and Analysis, \\ University of Nebraska, Lincoln, Nebraska 68588
}

\begin{abstract}
Relaxation processes in disordered nanostructures and spin glasses are investigated by examining the role of the magnetic anisotropy. To abstract from freezing processes associated with phase transitions, the consideration is restricted to a one-dimensional bond-disordered Ising spin glass. Using a Mattis-type gauge transformation, the spin glass is mapped onto a disordered ferromagnet. Based on the behavior of the spin chain we argue that spin-glass freezing is not necessarily related to a phase transition. The magnetic anisotropy, which is largely ignored in the Glauber approach to Ising dynamics, gives rise to cooperative deviations from the Arrhenius behavior and mimics Vogel-Fulcher relaxation. (C) 2001 American Institute of Physics. [DOI: 10.1063/1.1358337]
\end{abstract}

\section{INTRODUCTION}

For decades, relaxation processes in structural and magnetic glasses materials have attracted much attention in science and technology. A key problem is to express typical relaxation rates $\Gamma=1 / \tau$ as a function of temperature. In the early 1930s, it was recognized that the exponential Arrhenius law $\Gamma=\Gamma_{0} \exp \left(-E_{a} / k_{B} T\right)$ is of limited validity. An expression widely used in the field of structural glasses ${ }^{1-6}$ and spin glasses $^{5,7}$ is the phenomenological Vogel-Fulcher-Tamman (VFT) law

$$
\Gamma=\Gamma_{0} \exp \left(-\frac{E_{a}}{k_{B}\left(T-T_{\infty}\right)}\right) .
$$

Here, $E_{a}$ is an activation energy and $T_{\infty}$ is a temperature which, to some extent, epitomizes our knowledge about glasses. However, it remains an open question as to whether Eq. (1) describes a true phase transition.

The main aim of this article is to interpret magnetic relaxation processes in terms of the VFT equation. First, we discuss the effect of the magnetic anisotropy, and then we use a one-dimensional model to derive an explicit relaxationrate expression.

\section{SPIN-GLASS DYNAMICS AND ANISOTROPY}

A key problem is to what extent nonequilibrium approaches such as Glauber dynamics are able to provide an realistic description of magnetic materials. Glauber dynamics ${ }^{8}$ is defined by the master-equation transition rate

$$
W\left(s_{i} \rightarrow-s_{i}\right)=\frac{\Gamma_{0}}{2}\left(1-s_{i} \tanh \frac{h_{i}+\Sigma_{k} J_{i k} s_{k}}{k_{B} T}\right),
$$

where $h_{i}+\Sigma_{k} J_{i k} s_{k}$ is the interaction field acting on the $i$-th spin. It important to keep in mind that this expression is not able to account for the magnetic anisotropy of the glass: in a strict sense, the anisotropy of the Ising model is infinite, and the Glauber ansatz Eq. (2) overcomes this problem by introducing anisotropy-independent transition rates.

Figure 1 illustrates the freezing of the magnetization for

${ }^{a)}$ Electronic mail: rskomski@unlserve.unl.edu a disordered ferromagnet. Regions characterized by strong interatomic exchange coupling $J_{i k}$ (dark areas) block at comparatively high temperatures and remain largely immobile upon further cooling. The number $N$ of atoms per frozen block increases with decreasing temperature, and the energy barrier which needs to be overcome to reverse the magnetization scales of the block as $N K$, where $K$ is the anisotropy energy per atom. In terms of Eq. (1), this energy-barrier increase amounts to a VFT-like modification of the relaxationrate exponent. A three-dimensional example is ball-milled $\mathrm{Sm}_{2} \mathrm{Fe}_{17} \mathrm{~N}_{3}$ magnets. These nanostructures are randomanisotropy magnets and do not possess a ferromagnetic ground state, but their coercivity exceeds that of hard magnetic steels by more than two orders of magnitude, ${ }^{9}$ and equilibrium is not achieved for laboratory time scales.

\section{FREEZING}

To model the freezing process, we consider a onedimensional bond-disordered nearest-neighbor Ising spin glass (Edwards-Anderson spin glass) ${ }^{10-13}$ where the nearestneighbor exchange constants $J_{i j}$ obey $\left\langle J_{i j}\right\rangle=0$ and $\left\langle J_{i j}{ }^{2}\right\rangle$

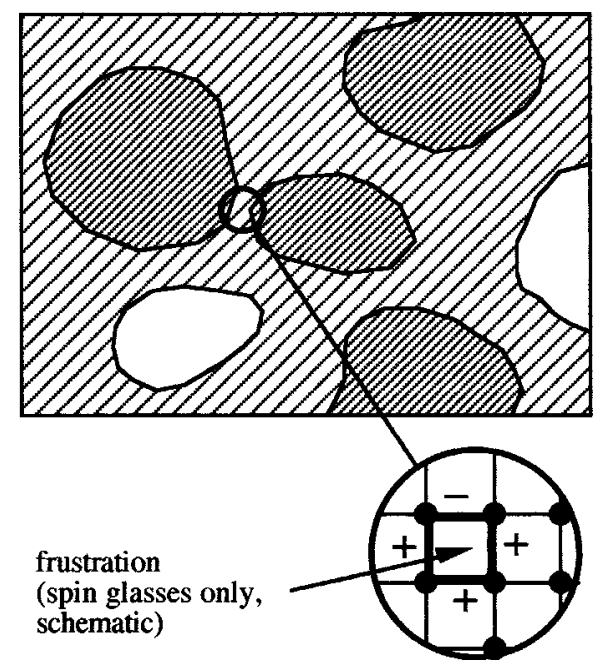

FIG. 1. Droplet picture of a disordered magnet. The darker the region, the stronger the local exchange. In the case of spin glasses, some of the bonds are frustrated (inset). 


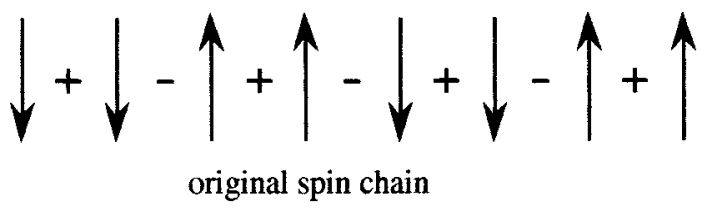

$\uparrow+\uparrow+\uparrow+\uparrow+\uparrow+\uparrow+\uparrow+\uparrow$

gauge-transformed spin chain

FIG. 2. Mattis-type gauge transformation for a spin chain. Open arrows indicate gauge-transformed spins.

$=J_{0}{ }^{2}$. To avoid the interference of finite-temperature phase transitions, we restrict the explicit calculations to a onedimensional model. First, we use a Mattis-type gauge transformation ${ }^{14}$ to transform the spin glass into a disordered ferromagnet. The transformation consists of multiplying each Ising spin variable $s_{i}= \pm 1$ by a variable $\epsilon_{i}= \pm 1$ so that $s_{i}{ }^{\prime}=\epsilon_{i} s_{i}, J_{i j} s_{i} s_{j}=J_{i j}{ }^{\prime} s_{i}{ }^{\prime} s_{j}{ }^{\prime}$, and $J_{i j}{ }^{\prime}=J_{i j} \epsilon_{i} \epsilon_{j}>0$. Choosing $\epsilon_{i}=-1$ transforms an antiferromagnetic bond into a ferromagnetic bond. The procedure, which is exact in one dimension, is illustrated in Fig. 2.

Since the gauge transformation does not change the bond strength, the resulting magnet is a spin chain with random ferromagnetic bonds. This situation corresponds to the droplet picture shown in Fig. 1. Let us assume that the magnitude of the exchange constants $J=\left|J_{i j}\right|$ obeys the exponential distribution

$$
P(J)=\frac{1}{J_{0}} \exp \left(-\frac{J}{J_{0}}\right) .
$$

On cooling the material from a very high temperature, the freezing process starts by strongly correlated spins in "dark" regions where $J$ is large. Figure 3 shows a realization of Eq. (3). At high temperatures, only the dark regions are coupled,

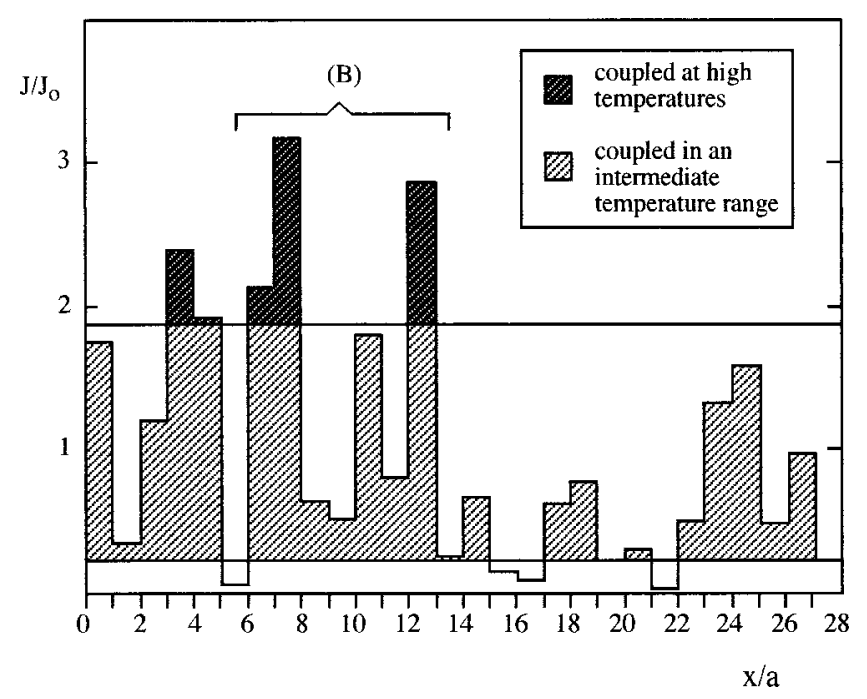

FIG. 3. Nearest-neighbor bond distribution. This is a one-dimensional ana$\log$ to Fig. 1.

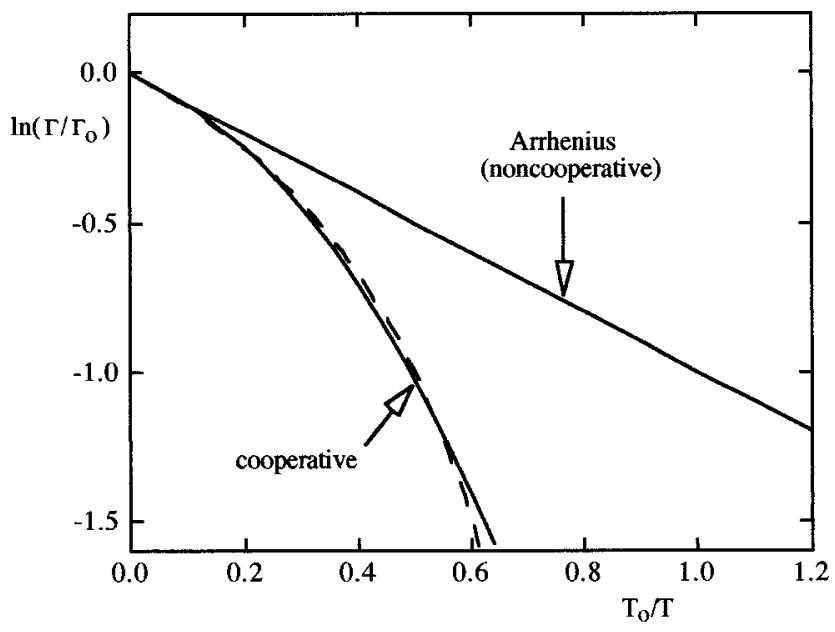

FIG. 4. Arrhenius plot of the relaxation rate.

but at low temperatures the spins form big droplets. Of course, the droplets do not exhibit ferromagnetism in a true sense: the magnetism of the spin glass is essentially superparamagnetic. However, the approach towards the superparamagnetic equilibrium may be very slow. For example, the block $(B)$ in Fig. 3 is only loosely exchange coupled to its neighborhood, but to realize the reversal it is necessary to switch 7 spins in a cooperative manner. This enhances the anisotropy energy, which must be overcome during reversal, by a factor of 7 .

To discuss this process in the Arrhenius diagram, we assume that all bonds $J>T$ are frozen and all bonds $J<T$ are free (easily broken by thermal excitation). For the distribution Eq. (2), the probability $p$ of encountering a free bond is then $1-\exp \left(-k_{B} T / J\right)$. On the other hand, the block size (the activation volume) $V=\mathrm{La}^{2}$ is equal to $1 / p$, so that the VFT term

$$
V_{\text {eff }}=\frac{V}{1-T_{0} / T},
$$

must be replaced by

$$
V_{\text {eff }}=\frac{V}{1-\exp \left(-k_{B} T / J\right)} .
$$

The asymptotics of Eq. (5) differ from that of Eq. (4), but for not-too-large time windows it is very difficult to distinguish between the two predictions. Figure 4 compares Eqs. (4) and (5) for $J=3 T_{0}$. We see that the two curves exhibit a pronounced deviation from the noncooperative Arrhenius behavior and are difficult to distinguish. It is interesting to note that the choice of the distribution $P(J)$, Eq. (3), is not very critical. For example, Gaussian and rectangular bond distributions yield results very similar to Fig. 4, although Eq. (5) is in general more complicated.

\section{DISCUSSION AND CONCLUSIONS}

Equation (5) shows that the cooperative deviations from the Arrhenius behavior do not necessarily correspond to a phase transition. The one-dimensional character of the spinglass model is of secondary importance in the present con- 
text, although the existence of related exact solutions ${ }^{13,15}$ encourages further research in this direction. Note that the gauge-transformed superparamagnetic droplets are difficult to polarize by a homogeneous magnetic field: they are most easily polarized by a random field $h_{i}{ }^{\prime}=\epsilon_{i} h_{i} \cdot{ }^{14,16}$ In a sense, the present approach is loosely related to the droplet approach by Fisher and Huse, ${ }^{17}$ although that theory starts from low temperatures.

Of course, the freezing mechanism investigated in this article is not the only mechanism giving rise to nonArrhenius relaxation: second-order phase transitions are accompanied by a critical slowing down $\left(\Gamma=0\right.$ at $\left.T_{c}\right)$ and a negative curvature in the Arrhenius diagram. However, finite-temperature phase transitions are restricted to two or more dimensions, and it is not possible to associate the cooperative behavior shown in Fig. 4 with a phase transition. Phase transitions are likely to enhance the non-Arrhenius character of the relaxation, as frustration in two- and multidimensional spin glasses may modify the relaxation behavior, but neither phase transitions nor frustration invalidate the disorder-related VFT mechanism introduced and examined in this work.

A challenge to future research is to provide a description going beyond the approximate result Eq. (5). In particular, for homogeneous materials, the dynamics has to reproduce results such as the " $25 k_{B} T$ ', magnetic-viscosity law for thermally activated magnetic reversal. ${ }^{18}$ This includes the exact dependence of $E_{a}$ on the anisotropy constant. (Here we have made the fair assumption that $E_{a}=K$ ).

In conclusion, we have developed a one-dimensional model which shows that exchange inhomogenities give rise to a cooperative glass transition at a temperature well above the (trivial) phase-transition temperature $T_{c}=0$. Our glasstransition picture is as follows. On cooling, gaugetransformed ferromagnetic droplets start to develop in regions with strong exchange, independently of whether the exchange is ferromagnetic or antiferromagnetic in real space and the corresponding freezing is a cooperative effect, involving the anisotropy energy of the interacting droplets.

\section{ACKNOWLEDGMENTS}

The authors are grateful to Prof. D. J. Sellmyer for valuable discussions. This research is supported by AFOSR and CMRA.

${ }^{1}$ H. Vogel, Phys. Z. 22, 645 (1921)

${ }^{2}$ G. S. Fulcher, J. Am. Ceram. Soc. 8, 339 (1925).

${ }^{3}$ G. Tamman and G. Hesse, Zeitschrift für anorganische und allgemeine Chemie 156, 245 (1926).

${ }^{4}$ E.-J. Donth, Glasübergang (Akademic, Berlin, 1981).

${ }^{5}$ K. Moorjani and J. M. D. Coey, Magnetic Glasses (Elsevier, Amsterdam, 1984).

${ }^{6}$ K. Binder, Eur. J. Phys. 20, 389 (1999).

${ }^{7}$ E. P. Wohlfarth, J. Phys. F: Met. Phys. 14, L155 (1984).

${ }^{8}$ R. J. Glauber, J. Math. Phys. 4, 294 (1963).

${ }^{9}$ R. Skomski, J. Magn. Magn. Mater. 157, 713 (1996).

${ }^{10}$ S. F. Edwards and P. W. Anderson, J. Phys. F: Met. Phys. 5, 965 (1975).

${ }^{11}$ D. Sherrington and S. Kirkpatrick, Phys. Rev. Lett. 35, 1792 (1975).

${ }^{12}$ M. Suzuki, Prog. Theor. Phys. 58, 1151 (1977).

${ }^{13}$ K. H. Fischer and J. A. Hertz, Spin Glasses (University Press, Cambridge 1991).

${ }^{14}$ D. C. Mattis, Phys. Lett. 56A, 421 (1976).

${ }^{15}$ G. Grinstein, A. N. Berker, J. Chalupa, and M. Wortis, Phys. Rev. Lett. 36, 1508 (1976).

${ }^{16}$ I. Morgenstern, K. Binder, and R. M. Hornreich, Phys. Rev. B 23, 287 (1981).

${ }^{17}$ D. S. Fisher and D. A. Huse, Phys. Rev. Lett. 56, 1601 (1986).

${ }^{18}$ R. Skomski and J. M. D. Coey, Permanent Magnetism (Institute of Physics, Bristol, 1999). 